\title{
New developments in scanning microelectrochemical techniques: a highly sensitive route to evaluate degradation reactions and protection methods with chemical selectivity
}

Ricardo M. Souto ${ }^{1}$, Daniel Filotás ${ }^{2,3}$, Bibiana M. Fernández-Pérez ${ }^{1}$, Lívia Nagy ${ }^{2,3}$, and Géza Nagy ${ }^{2,3}$

${ }^{1}$ Department of Chemistry, University of La Laguna, P. O. Box 456, 38200 La Laguna (Tenerife), Spain

2 János Szentágothai Research Center, University of Pécs, Ifjúság útja 20, 7624 Pécs, Hungary

${ }^{3}$ Department of General and Physical Chemistry, Faculty of Sciences, University of Pécs, Ifjúság útja 6, 7624 Pécs, Hungary

\begin{abstract}
.
The scanning electrochemical microscope (SECM) offers a highly sensitive route to evaluate degradation reactions and protection methods with chemical selectivity by using ion-selective microelectrodes as tips, thus operating SECM potentiometrically. Spatially resolved imaging of electrochemical reactivity related to each component of the investigated material can thus be effectively monitored selectively both in situ and in real time. The applicability of this method has been illustrated using two practical examples of galvanic corrosion processes, namely a model Zn$\mathrm{Cu}$ pair, and a metal-coating system consisting in the exposure of cut edges of coil-coated galvanized steel to aqueous saline environment. In this contribution, localized $\mathrm{pH}$ and zinc(II) ion distributions originated around the corroding systems immersed in $1 \mathrm{mM} \mathrm{NaCl}$ solution are shown.
\end{abstract}

Keywords: Scanning electrochemical microscopy; Corrosion; ion-selective microelectrode tips; Metal-coating systems; pH distributions; concentration distributions. 


\section{Introduction}

Corrosion is an electrochemical phenomenon with enormous economic influence. It is the gradual degradation of elemental metals converting them to their more stable oxidized forms. Hence the investigation of corroding systems and understanding complex corrosion processes are of major interest as to develop more efficient corrosion protection methods. Though corrosion processes may present different mechanisms and reactions, they all share the common electrochemical nature of the process. Thus, electrochemical techniques are usually employed for their investigation. In addition to conventional electrochemical techniques, scanning microelectrochemical methods are becoming essentials tools for corrosion and materials science scientists as they provide in situ localized chemical information of the sample of interest with high resolution [1].

To electrochemically image a material surface in an aqueous solution as it experiences chemical activity, methods such as the Scanning Vibrating Electrode Technique (SVET) and the Scanning Electrochemical Microscopy (SECM) are microelectrochemical techniques which can decipher local reaction sites. In these techniques, a microelectrode (ME), also known as the tip, and substrate are positioned within an electrochemical cell along with a reference and counter electrodes. The tip is capable of moving in $X-Y-Z$ coordinates, and thus can raster the substrate surface at any prescribed height. In SVET localized potential gradients of the sample surface caused by a local ionic current occurring around anodic and cathodic areas are detected with a respect to values obtained in the bulk solution away from the sample [2]. Although this technique cannot identify the precise composition of the sensed species, it is a very efficient way to characterize corroding systems. SECM uses a polarizable working electrode instead, thus effectively measuring currents. The current recorded by the tip is a function of the local solution composition, the tip-substrate distance and the electrochemical/electrical activity of the substrate. An image of the spatial electrochemical reactions on a sample can thus be obtained by recording/analysing the tip currents, as the tip is rastered across the substrate surface. This is because currents associated with the electrochemical reaction at the tip, experience perturbations when in close proximity with the substrate, therefore enabling one to record a chemical micrograph of the surface. Alternately to amperometric operation, ion-selective microelectrodes can also be employed as tips, thus allowing the selective monitoring of different ionic species. Among them, Sb-based microelectrodes can be operated as amperometric/potentiometric probes, thus effectively sensing faradaic currents associated to electron transfer reactions, as well as to selectively image $\mathrm{pH}$ distributions [3]. Finally, ion-selective microelectrodes (ISME) can be employed to monitor concentrations of a selected chemical species [4].

In this contribution we report new developments in scanning electrochemical microscopy based on the application of potentiometric operation for the chemical sensing of corroding materials and 
their protection, using a model Zn-Cu galvanic pair and a cut edge of painted galvanized steel exposed to aqueous solution to illustrate the chemical resolution of the technique.

\section{Experimental}

SECM experiments were performed using an instrument manufactured by Sensolytics and operated with an Autolab bipotentiostat provided with a frequency response analyzer, under computer control. SECM measurements were performed on a model $\mathrm{Zn}-\mathrm{Cu}$ galvanic pair and on cut edges of coil-coated galvanized mild steel (CCS) immersed in $1 \mathrm{mM} \mathrm{NaCl}$ solution. The Zn-Cu galvanic pair was prepared by embedding wires of $1 \mathrm{~mm}$ dia. into epoxy resin sleeve with $1 \mathrm{~mm}$ separation between them. Surface finish of the top side comprised wet grinding with emery papers of 800-4000 grit size, followed by polishing with alumina slurries of $0.3 \mu \mathrm{m}$ particle size. Electrical contact between the metals was performed at the rear of the sample. The mild steel foil of the CCS was $400 \mu \mathrm{m}$ thick, whereas the galvanized layers applied at both sides were ca. $30 \mu \mathrm{m}$ thick, and coated with a $5 \mu \mathrm{m}$ polyester primer containing strontium chromate as inhibitor. One of the sides was further coated with a $20 \mu \mathrm{m}$ white polyester topcoat containing $\mathrm{TiO}_{2}$. This system was embedded in epoxy resin. The exposed cut edge was ground with SiC papers down to 4000 grit, and polished with $0.5 \mu \mathrm{m}$ alumina slurries. Non painted galvanized steel cut edges were prepared by removing the paint layers with acetone. Samples were surrounded laterally by Sellotape to contain ca. 3mL of the test solution.

SECM probes were fabricated from pulled borosilicate capillaries to build micropipettes that will contain the sensing element after silanization by soaking in $5 \%$ dichloro-dimethyl-silane in $\mathrm{CCl}_{4}$ solution, and subsequently dried at $120{ }^{\circ} \mathrm{C}$ for $30 \mathrm{~min}$. The preparation of the Zn ISME electrode involved electrochemical polymerization of PEDOT on a $33 \mu \mathrm{m}$ dia. carbon fiber [4]. A small amount of Zn-ion selective cocktail was allocated into the micropipette tip, solvent evaporated for about 24 $\mathrm{h}$, before the PEDOT-coated carbon fiber was inserted in the lumen of the capillary. In this way, the end of the carbon fiber was immersed in the ion-selective cocktail. $\mathrm{pH}$ measurements were made using an Sb microelectrode built by melting Sb powder inside the borosilicate capillary [4] . The electric contact to the resulting antimony fiber was attained by introducing a copper wire and liquid mercury.

\section{Results and discussion}

By using the antimony disc electrode, $\mathrm{pH}$ distributions were monitored over the exposed cut edge. Measurements could be recorded as 1-D scan lines, where the sensing probe is moved either exclusively in the $Z$ axis, along an imaginary line drawn towards the center of the sample (Z-approach curves), or in the $X$ axis, i.e., along an imaginary line drawn perpendicular to the sample. In the latter, the time evolution of the system can be followed in real time due to the rather slow acquisition time 
of the technique. Alternately, 2-D maps can also be recorded; that is, the sensing probe is scanned in both $X$ and $Y$ directions although scanning relatively big samples as those described in this work may eventually take a long time in comparison to the rate of the corrosion. Since high scan rates cause poor resolution, and distortion, they can be selected at certain times in order to obtain an estimate of the behaviour for the complete exposed system.

Potentiometric SECM imaging of the model Zn-Cu galvanic pair immersed in $1 \mathrm{mM} \mathrm{NaCl}$ solution was first performed by moving the dual microelectrode probe relative to the sample placed horizontally at the bottom of the small electrochemical cell. Concentration distributions of zinc and hydrogen ions in the electrolyte adjacent to the two metal samples were recorded using two different procedures, namely as a function of either the distance to the metal substrate (i.e., $Z$-approach curves), or the location on the sample while keeping fixed the probe-sample distance (e.g, by moving the probe parallel to the surface). The latter allows a spatially-resolved image of the dynamic corrosion process to be obtained in situ and to follow its evolution with time. On the other hand, Z-approach curves will facilitate adequate positioning of the probe relative to the sample and selection of the optimum operating distance for 2D imaging, as well as to visualize the release/consumption and the diffusion of the participating species to/from the bulk solution. Typical $Z$-approach curves times over the centres of the $\mathrm{Zn}$ and $\mathrm{Cu}$ wires that were registered at various elapsed times in the $1 \mathrm{mM} \mathrm{NaCl}$ solution are shown in Fig. 1A-B. These curves were obtained by transforming the recorded electrode potential values to either $\mathrm{pZn}$ or $\mathrm{pH}$ values using calibration data. The $\mathrm{pZn}-d$ curves in Fig. 1A clearly display zinc ion concentration to be highest at the surface of the metal, and it decreases linearly with distance from the metal surface until the signal recorded at the Zn-ISME probe becomes almost invariant for sufficiently long distances from the surface, certainly with values beyond the linear calibration interval of the probe. More interestingly, the progress of the corrosion reaction can be roughly followed from the time dependence of the $Z$-approach curves. That is, there is a first time interval related to the onset of $\mathrm{Zn}$ dissolution from the surface, in which the surface becomes progressively deactivated after about $40 \mathrm{~min}$, a trend that is evidenced by a sustained decrease in the Zn-ion concentrations for any given distance from the metal surface. Conversely, longer exposures reveal a vigorous reactivation of the zinc strip towards metal dissolution, leading to the highest zincion concentrations recorded in this experiment. Therefore, time resolution is a key factor in addition to space-resolution for extracting the most information possible in a microelectrochemical characterization of the highly dynamic corrosion processes.

The evolution of a dynamic process such as corrosion reactions can be followed by recording scan lines that pass over the centres of the two metals for a geometry such as the Zn-Cu system considered herein, thus enabling the acquisition of sufficient data to analyze the system evolution. In this way, $\mathrm{pH}$ distributions such as those displayed in Fig. 1C can be attained, where the rather homogeneous 
distribution of the cathodic activity over the copper strip is observed at various distances from the metal surface in terms of local alkalization of the electrolyte.

Analogously, Fig. 2 depicts line scans taken at different exposure times either for the galvanized steel system without paint layers at either side (Fig. 2A) and for the galvanized steel system with the two paint layers (Fig. 2B). In general, they show an alkaline region developing directly above the steel matrix, where the reduction of oxygen to hydroxide ions occurs as the cathodic half reaction. The $\mathrm{pH}$ changes decrease with time for both materials due to the precipitation of corrosion products on the surface of the material, effectively blocking portions of the metallic surface from contact with the environment. Conversely, local acidification develops above the zinc strips as result of metal dissolution and subsequent hydrolysis in the aqueous phase, a reaction that occurs with the release of hydrogen ions. The different chemical activity of the coated and the bare galvanized steel cut edges is also readily observable by comparing the shape of the $\mathrm{pH}$ distributions recorded for each system. Rather symmetrical $\mathrm{pH}$ distributions were obtained in the case of non-coated galvanized steel, with both zinc strips equally acting as sacrificial anodes. On the contrary, the $\mathrm{pH}$ distributions developing on the painted galvanized steel are notoriously asymmetrical, clearly reflecting the different composition of the paint layers applied at either side. In this way, the corrosion inhibition effect of strontium chromate contained in the polymer films leads to a smaller rate of metal dissolution, and subsequent decrease in acidification. In addition, the thicker polymer layer at the left further protects the adjacent zinc compared to the layer at the right side, which dissolves preferentially.

Analogously, the $\mathrm{Zn}^{2+}$ selective electrode was employed to measure the local distributions of $\mathrm{Zn}^{2+}$ concentration over the cut edge systems, and scan lines recorded at different exposure times are shown in Fig.4. At the beginning of the experiment, the concentration of $\mathrm{Zn(II)}$ ions increases up to 50-70 min, and it is followed by a decrease at longer times. This is an indication of the onset of corrosion products precipitation as result when the soluble zinc ions encounter a more alkaline environment. The different behaviour of the painted and bare galvanized steels is also observed here, with preferential dissolution of one zinc layer in the case of the painted system shown at the right.

Examples of 2-D maps are shown in Fig. 4. They were measured after recording the scan lines in Figs. 2 and 3, thus giving an image of the resulting surface of the materials. They agree well with the description derived from the line scans, i.e., more symmetrical distributions for pZn on the nonpainted galvanized steel cut edge compared to the painted system. Yet, the protective effect of the paint layers is evidenced by the lower pZn values attained by the non-coated sample.

\section{Conclusions}

Scanning electrochemical microscopy (SECM) is a highly sensitive tool for the characterization of surface reactivity of materials as it provides images with chemical and spatial resolution. The 
potential of potentiometric operation for the in situ monitoring of corrosion processes and the efficiency of protection methods has been shown. Both scan line and 2-D maps offer complementary information on the actual mechanisms operating in the system and their time evolution. Ion-selective microelectrodes are used as sensing probes in this case.

\section{Acknowledgements}

D. Filotás acknowledges an ERASMUS+ mobility grant to the University of La Laguna, and B.M.F.-P. thanks the Canary Agency for Research, Innovation and Information Society and the European Social Fund for a research contract. Financial support by the Spanish Ministry of Economy and Competitiveness (MINECO, Madrid, Spain) and the European Regional Development Fund (Brussels, Belgium) under grant CTQ2016-80522-P is also acknowledged.

\section{References}

[1] M.B. Jensen, D.E. Tallman, Application of SECM to corrosion studies, in: AJ Bard, and CG Zoski (Eds.), Electroanalytical Chemistry: A Series of Advances, Vol. 24, CRC Press, Boca Raton, 2012, pp. 171-286.

[2] S. Rossi, M. Fedel, F. Deflorian, M.C. Vadillo, Localized electrochemical techniques: Theory and practical examples in corrosion studies, C. R. Chim. 11 (2008) 984-994.

[3] J. Izquierdo, L. Nagy, Á. Varga, J.J. Santana, G. Nagy, R.M. Souto, Spatially resolved measurement of electrochemical activity and $\mathrm{pH}$ distributions in corrosion processes by scanning electrochemical microscopy using antimony microelectrode tips, Electrochim. Acta 56 (2011) 88468850 .

[4] R.M. Souto, J. Izquierdo, J.J. Santana, A. Kiss, L. Nagy, G. Nagy, Progress in scanning electrochemical microscopy by coupling potentiometric and amperometric measurement modes, in: A. Méndez-Vilas (Ed.), Current Microscopy Contributions to Advances in Science and Technology, Formatex Research Center, Badajoz, 2012, 1407-1415. 

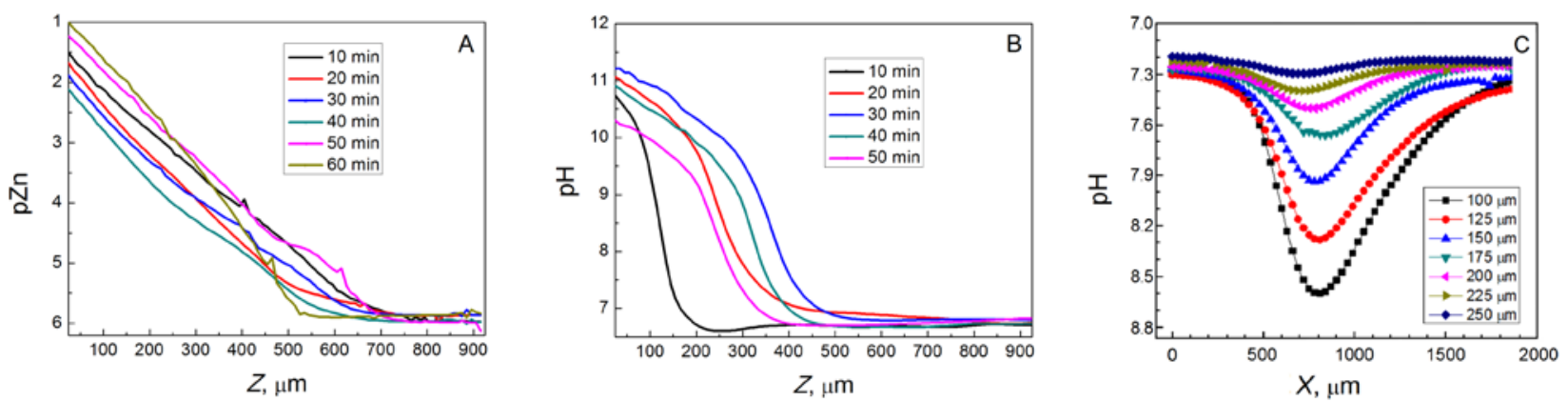

Figure 1. Potentiometric SECM line scans recorded over the $\mathrm{Zn}-\mathrm{Cu}$ galvanic couple in $1 \mathrm{mM} \mathrm{NaCl}$ using the individual probes of the double-barrel microelectrode. (A, B) Z-approach curves to a Zn$\mathrm{Cu}$ galvanic couple in $1 \mathrm{mM} \mathrm{NaCl}$ recorded using the individual probes of the double-barrel microelectrode at different immersion times as indicated. $Z$-approach curves giving (A) the $\mathrm{Zn}^{2+}$ ion distribution above the $\mathrm{Zn}$ sample, and (B) the $\mathrm{pH}$ distribution above the $\mathrm{Cu}$ sample as a function of time; and (C) $\mathrm{pH}$ distribution above the Cu sample at various tip-substrate distances.
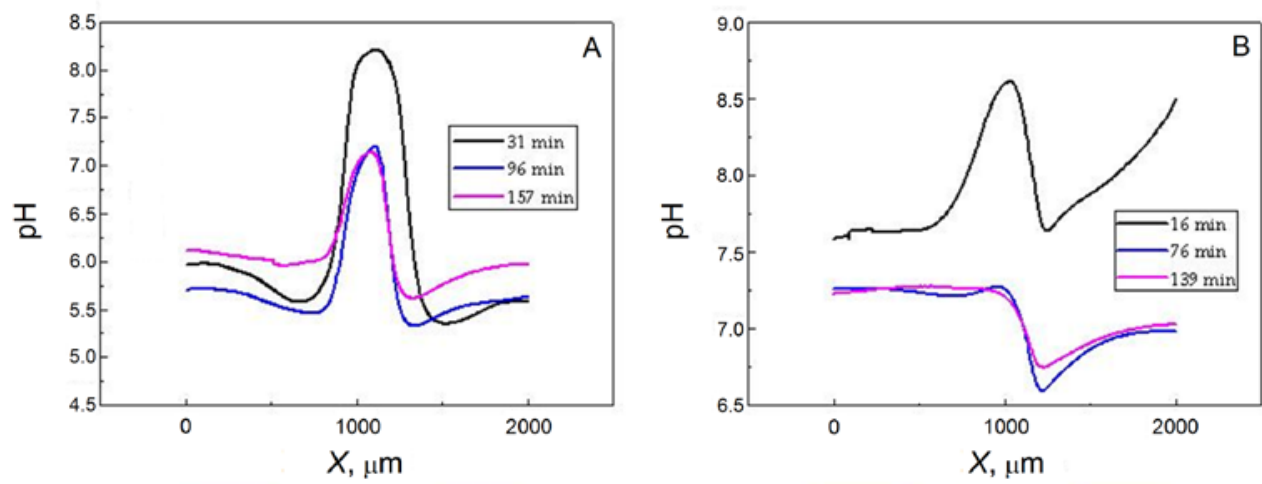

Figure 2. Line scans recorded with the $\mathrm{pH}$ sensitive Sb-ME above the cut edges of: (A) non-painted galvanized steel, and (B) painted galvanized steel, in $1 \mathrm{mM} \mathrm{NaCl}$ solution after various times.
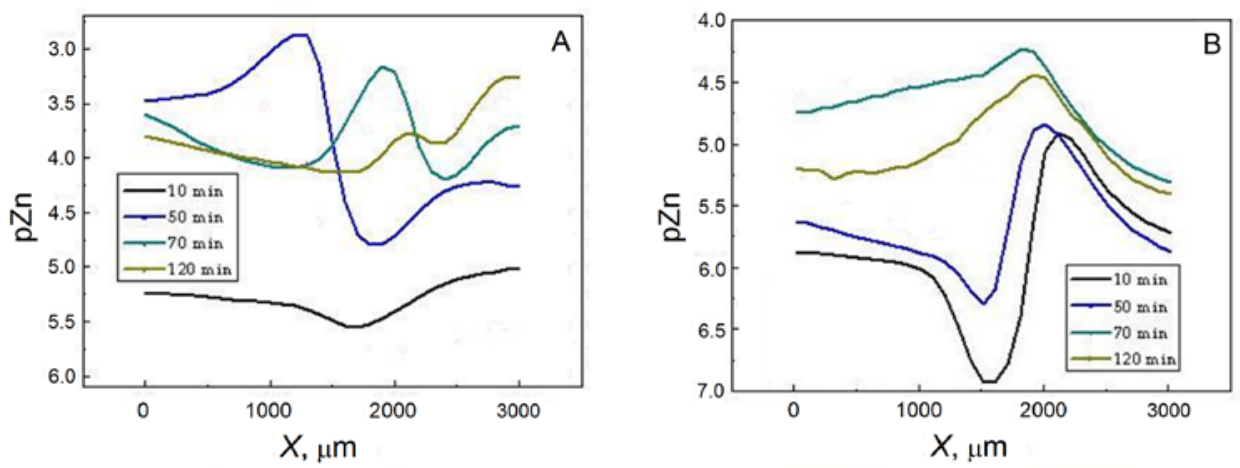

Figure 3. Line scans recorded with the Zn-ISME above the cut edges of: (A) non-painted galvanized steel, and (B) painted galvanized steel in $1 \mathrm{mM} \mathrm{NaCl}$ solution for the times indicated in the graphs. 

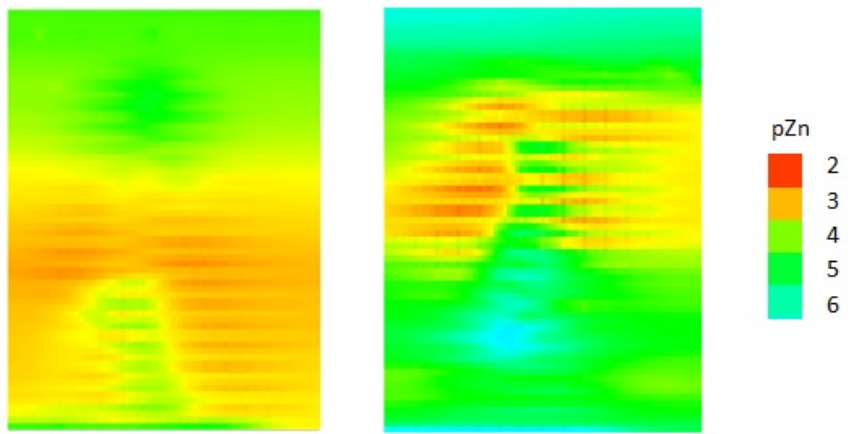

Figure 4. $\mathrm{Zn}^{2+}$ ion distribution maps recorded above the cut edges of: (A) non-painted, and (B) painted galvanized steel in $1 \mathrm{mM} \mathrm{NaCl}$ solution. The images correspond to $2000 \mu \mathrm{m} \times 6000 \mu \mathrm{m}$ in $X$ and $Y$. 